\title{
Real-time optical demultiplexing with the chirped pulses
}

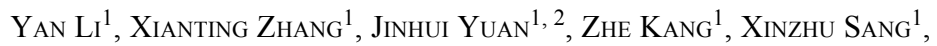

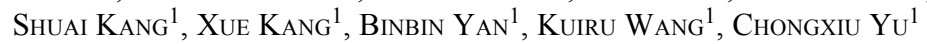 \\ ${ }^{1}$ State Key Laboratory of Information Photonics and Optical Communications, \\ Beijing University of Posts and Telecommunications, \\ P.O. Box 72 (BUPT), 100876, Beijing, China \\ ${ }^{2}$ Photonics Research Center, Department of Electronic and Information Engineering, \\ Hong Kong Polytechnic University, Hung Hom, Kowloon, Hong Kong, China
}

\begin{abstract}
A scheme for real-time optical demultiplexing is proposed by utilizing the time-broadened and linearly chirped pulses instead of the conventional mode-locked pulses. The copies of the optical -time-division-multiplexed signal are acquired through a dual-pump parametric gate and used as the parametric multicast block. Simulation results show that the demultiplexing from $160 \mathrm{~Gb} / \mathrm{s}$ down to sixteen $10 \mathrm{~Gb} / \mathrm{s}$ tributaries can be achieved only by using a sampling source of $10 \mathrm{GHz}$. The proposed scheme can effectively reduce the complexity of parallel processing, and find important applications in the high-speed all-optical signal processing.
\end{abstract}

Keywords: linearly chirped pulse, parametric process, real-time optical demultiplexing.

\section{Introduction}

The capacity of current communication system has been motivated by the increasing capacity and granularity to meet the urgent requirement of the users. The increase in the capacity of optical communication system supplies the high-speed data service to support various professional works, such as high-definition video, three-dimensional video transmission, radar system, and so on. Some schemes have been proposed to achieve the high-speed signal processing [1- $\underline{4}]$. Unfortunately, the inherent electronic bottleneck imposed by the electrical devices in the previous schemes greatly limits the speed of signal processing. Recently, all-optical signal processing has been considered as a good candidate to circumvent the electronic limitation, and widely used in the high -speed signal processing [ $\underline{5}-\underline{8}]$.

Optical time division multiplexing (OTDM) is usually used for the high-speed data generation beyond the bandwidth limitation of electronics. However, because some demultiplexing schemes proposed for OTDM [ $\underline{9}-\underline{13}]$ only produce the single tributary 
at some time, the complexity of processor is increased, and the corresponding scalability is greatly decreased. In order to remove these limitations, a new pre-processing architecture, which is called as multicast parametric synchronous sampling (MPASS), is recently demonstrated [14-16], whose operation principle is based on the parametric multicasting (PM), synchronization (PS), and sampling in a parametric gate (PG). Compared to the multicasting architecture based on the single pump, although a dual-pump fiber optical parametric amplifier (FOPA) with a higher conversation efficiency can reduce the rate of sampling source, the architecture becomes more complicated as the pump number is increased, and the synchronism of two pumps needs to be strictly controlled $[16,17]$. An alternative scheme should be used to address the problem. A scheme of multicast parametric synchronous sampling is preliminarily proposed for reducing the requirement of the copy number in our previous work [18], where the chirped pulses are used instead of the conventional mode-lock pulses. However, the scheme for the multicast parametric synchronous sampling can only realize the sampling rate of $120 \mathrm{GSa} / \mathrm{s}$ by a $10 \mathrm{GHz}$ sampling source. In this paper, we propose a novel scheme for retrieving all sixteen $10 \mathrm{~Gb} / \mathrm{s}$ tributaries of a $160 \mathrm{~Gb} / \mathrm{s}$ OTDM signal. The time-broadened and linearly chirped pulses are utilized to realize the real-time optical demultiplexing. Simulation results demonstrate the good performance and high feasibility of the proposed scheme.

\section{Theory and operation principle}

For the signal multicast, a dual-pump FOPA is used to achieve the more copies. The generation of four-wave mixing (FWM) involved in two pump waves can act as the new set of pumps $[\underline{14}-17,19,20]$. The generated pumps can be used as self-seeded pumps, which play an important role in the wavelength multicasting of high speed signals. The combination of degenerate and non-degenerate FWM processes can generate eight other idler waves. The physical principle is shown in Fig. 1. The original pumps (pump 1 and pump 2) are located at $\omega_{1}$ and $\omega_{2}, \Delta \omega$ is referred to as the frequency spacing from the pump 1 to the pump 2, and the signal wave at $\omega_{\mathrm{s}}$ separated from the pump 1 by $\omega=\omega_{\mathrm{s}}-\omega_{1}$. In the propagation process of the signal wave, three side-bands are pro-

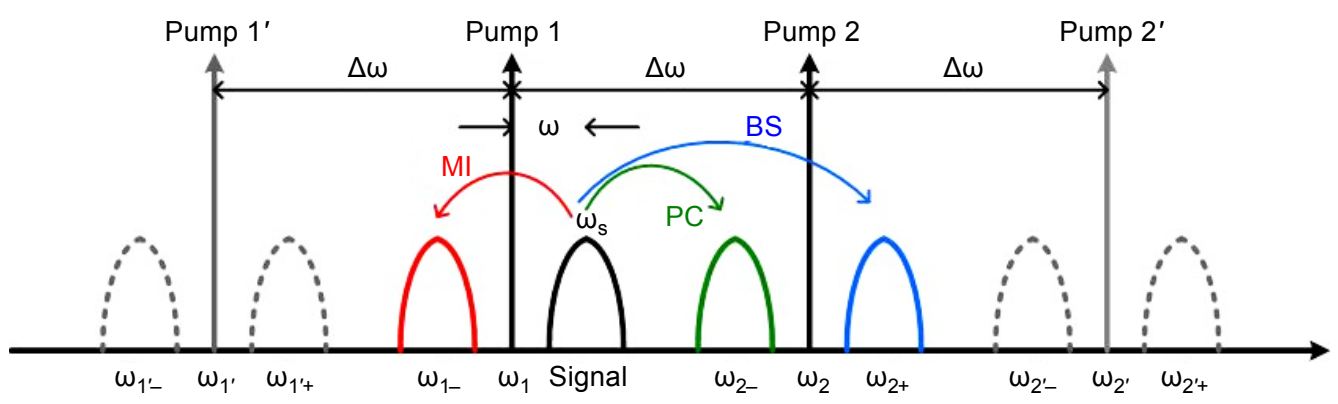

Fig. 1. FWM driven by two-pump self-seeding process. 


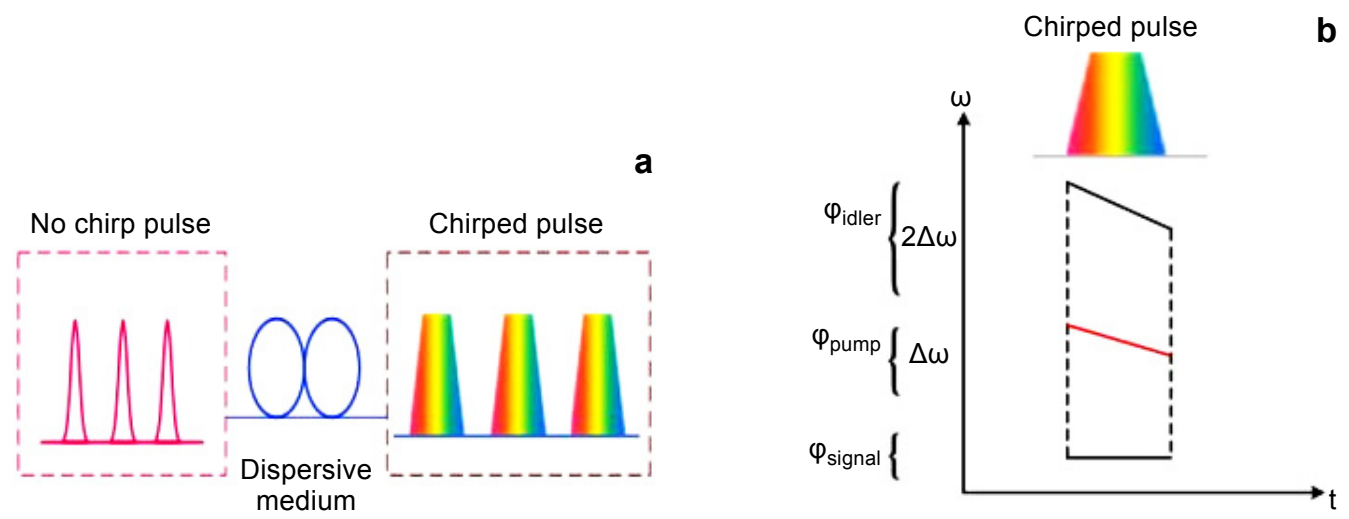

Fig. 2. Operation principle of obtaining the linear chirp via a dispersive medium (a). Chirp diagram of FWM-based optical sampling (b).

duced by the interaction with pump waves $[\underline{16}, \underline{19}, \underline{21}]$. The modulation instability (MI) produces a idler band with the frequency $\omega_{1-}=\omega_{1}-\omega$, the phase conjugation (PC) produces a idler band with the frequency $\omega_{2-}=\omega_{2}-\omega$, and the Bragg scattering (BS) produces a idler band with the frequency $\omega_{2+}=\omega_{2}+\omega$. The original pumps generate the new set of pumps (pump 1' and pump $2^{\prime}$ ) centered at $\omega_{1^{\prime}}$ and $\omega_{2^{\prime}}$, where $\omega_{1^{\prime}}=\omega_{1}-\Delta \omega$ and $\omega_{2^{\prime}}=\omega_{2}+\Delta \omega$, and the power equalization over the four pump tones is accomplished by the phase-matching condition. The additional four idler bands generated at $\omega_{1^{\prime}-}=\omega_{1^{\prime}}-\omega, \omega_{1^{\prime+}}=\omega_{1^{\prime}}+\omega, \omega_{2^{\prime}-}=\omega_{2^{\prime}}-\omega, \omega_{2^{\prime}+}=\omega_{2^{\prime}}+\omega$, respectively. Therefore, the signal is copied to multiple spectral replicas at $\omega_{1^{\prime}-}, \omega_{1^{\prime}+}, \omega_{1-}, \omega_{2-}, \omega_{2+}, \omega_{2^{\prime}-}, \omega_{2^{\prime}+}$. The response over the multicast copies can be further equalized by equalization of the new pumps [20].

For the demultiplexing process of signal, the linear chirped pulses are used as the pump, which can be obtained via a dispersive fiber, as shown in Fig. 2a. When the pulses are propagated inside the dispersive fiber, the dependence of the pulse phase on the time can be described by [22]]:

$$
\varphi_{\mathrm{p}}(z, T)=-\frac{\operatorname{sgn}\left(\beta_{2}\right)\left(z / L_{\mathrm{D}}\right)}{1+\left(z / L_{\mathrm{D}}\right)^{2}} \frac{T^{2}}{2 T_{0}^{2}}+\frac{1}{2} \tan ^{-1}\left(z / L_{\mathrm{D}}\right)
$$

where $T_{0}$ is the initial width at half maximum (FWHM), $L_{\mathrm{D}}$ and $\beta_{2}$ are the dispersion length and the group velocity dispersion of the fiber, respectively. The time-broadened pulses are used as the sampling pulses, and delivered into a span of highly nonlinear fiber (HNLF). During the parametric process $[\underline{21}, \underline{23}, \underline{24}]$, the chirped pulses will modulate the phase of the idler signal as $A_{\mathrm{i}}(t) \propto A_{\mathrm{p}}^{2}(t) A_{\mathrm{s}, n}^{*}(t)$, where $A_{\mathrm{i}}(t), A_{\mathrm{p}}(t)$, and $A_{\mathrm{s}, n}(t)(n=1,2, \ldots, 8)$ are the idler signal, the pump pulses, and the copies, respectively. Thus, the phase of the idler signal is as following:

$$
\varphi_{\mathrm{i}}(z, T)=2 \varphi_{\mathrm{p}}(z, T)-\varphi_{\mathrm{s}, n}(z, T)
$$


Compared to the chirp of the pump, the chirp of the signal copies can be neglected. So the chirp $\delta \omega_{\mathrm{i}}$ is only the time derivative:

$$
\delta \omega_{\mathrm{i}}(T)=-2 \frac{\partial \varphi_{\mathrm{p}}}{\partial T}=\frac{2 \operatorname{sgn}\left(\beta_{2}\right)\left(z / L_{\mathrm{D}}\right)}{1+\left(z / L_{\mathrm{D}}\right)^{2}} \frac{T}{T_{0}^{2}}
$$

Equation (3) indicates that the chirp of the idler wave is twice as that of the pump, as shown in Fig. 2b. The time demultiplexing points can be extracted by filtering out different frequencies of the idler waves.

In the demultiplexing block, the serial-to-parallel conversion rate is as following:

$$
R_{\mathrm{S}}=\frac{\left|\delta \omega_{\mathrm{i}}(T)\right|_{\max } n R_{\mathrm{pump}}}{\Delta f}
$$

where $n$ is the number of copies, $R_{\text {pump }}$ is the repetition rate of the pump, and $\Delta f$ is the bandwidth of filters. If $\Delta f$ is a constant, a large chirp of the idler waves can support a higher serial-to-parallel conversion rate. Therefore, the time demultiplexing points can be extracted by filtering out different frequencies of the idler waves, and the proposed scheme has the great potential to sample the original signal with a pump source, whose sampling rate is far below the Nyquist rate.

The operation principle of real-time parametric processor includes copying the original signals, synchronization of the signal copies on time, and demultiplexing of all copies by the chirped pulses. The architecture of the parametric processor is shown in Fig. 3. The return-to-zero (RZ) OTDM data stream at an input wavelength $\lambda$ is copied to eight spectral replicas $\left(\lambda_{1}, \lambda_{2}, \ldots, \lambda_{N}\right)$ by a wavelength multicasting block, and these copies are delayed by a delay block with the temporally delay $\Delta \tau$ defined as $2 / R$, where $R$ is the rate of the OTDM signal. The delayed copies are sent to the parametric demultiplexing block, where the linearly chirped pulses are used as the sampling pulses instead of the conventional mode-locked pulses. The parametric interaction between the $N$ copies and the sampling pulses generates the $N$ sampled idlers. Finally, the samples are separated by using a wavelength demultiplexer, which results in continuously streaming substrate data output. Then, one $160 \mathrm{~Gb} / \mathrm{s}$ OTDM signal can be demultiplexed to sixteen $10 \mathrm{~Gb} / \mathrm{s}$ tributaries.

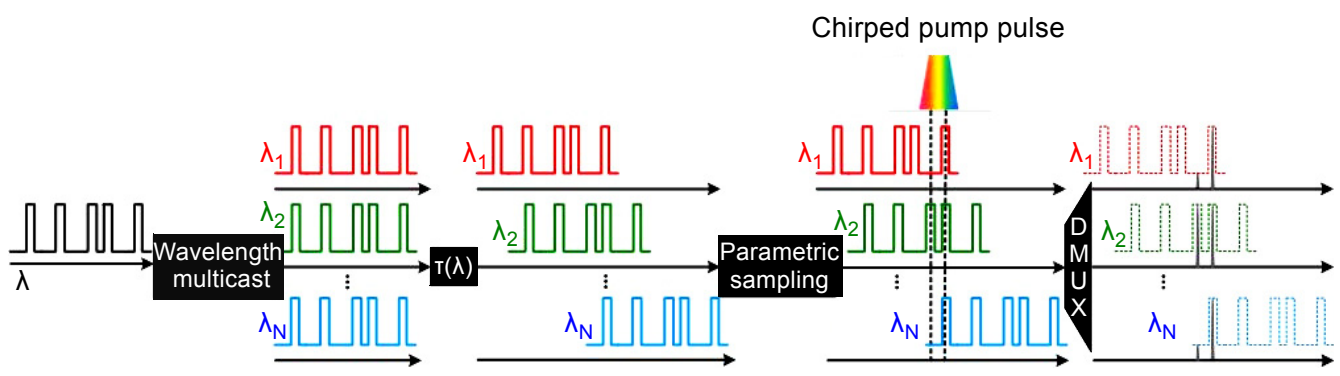

Fig. 3. Multicast parametric synchronous optical demultiplexing. 


\section{Results and discussion}

The simulation set-up of the proposed scheme is shown in Fig. 4, which consists of the parametric multicast block, time delay block, and demultiplexing block. In the parametric multicasting block, two pump waves centered at 1536.2 and $1553.0 \mathrm{~nm}$ (pump 1 and pump 2) are coupled into a wavelength division multiplexer (WDM), and delivered to another one. The $160 \mathrm{~Gb} / \mathrm{s}$ optical-time-division-multiplexed (OTDM) data signal is generated at $1540.5 \mathrm{~nm}$. The OTDM signal is amplified by an erbium-doped optical fiber amplifier (EDFA 1), and filtered by an optical band-pass filter (BPF 1) to reject the amplified spontaneous emission (ASE). The OTDM signal and the two pumps are launched into a 90-m-long highly nonlinear fiber (HNLF 1), whose attenuation coefficient is $0.2 \mathrm{~dB} / \mathrm{km}$, zero dispersion wavelength (ZDW) is $1540.0 \mathrm{~nm}$, and dispersion slope is $0.03 \mathrm{ps} /\left(\mathrm{nm}^{2} \mathrm{~km}\right)$. In the time delay block, a temporal delay generated by a delayer is adjusted on each copy. In the demultiplexing block, a $10 \mathrm{GHz}$ Gaussian pulse centered at $1585.0 \mathrm{~nm}$ is used. The pulse train propagates in a $5-\mathrm{km}$-long standard single-mode fiber (SSMF) to obtain the linear chirp. The ZDW and dispersion slope of the SSMF are $1583.7 \mathrm{~nm}$ and $0.027 \mathrm{ps} /\left(\mathrm{nm}^{2} \mathrm{~km}\right)$, respectively. The Gaussian pulses are combined with eight copies prior to a 20-m-long HNLF 2, which has the attenuation coefficient of $0.2 \mathrm{~dB} / \mathrm{km}$ and a dispersion slope of $0.027 \mathrm{ps} /\left(\mathrm{nm}^{2} \mathrm{~km}\right)$. The spectral distinct samples are separated by an arrayed waveguide grating (AWG). Rigorous bit-error-rate (BER) measurements are carried out by extracting all the tributaries from each one of the eight idlers.

In the gain regime of the parametric device, the pump noise will be amplified, and transferred to the copies. In order to circumvent the effect, two continuous waves (CWs) are used as the pump waves for multicasting [14, 19]. The pump 1 and pump 2 centered at 1536.2 and $1553.0 \mathrm{~nm}$ are delivered to the HNLF 1 along with a $160 \mathrm{~Gb} / \mathrm{s}$ OTDM signal at $1540.5 \mathrm{~nm}$. The pump 1 and pump 2 powers are chosen as 1.2 and $0.6 \mathrm{~W}$, re-

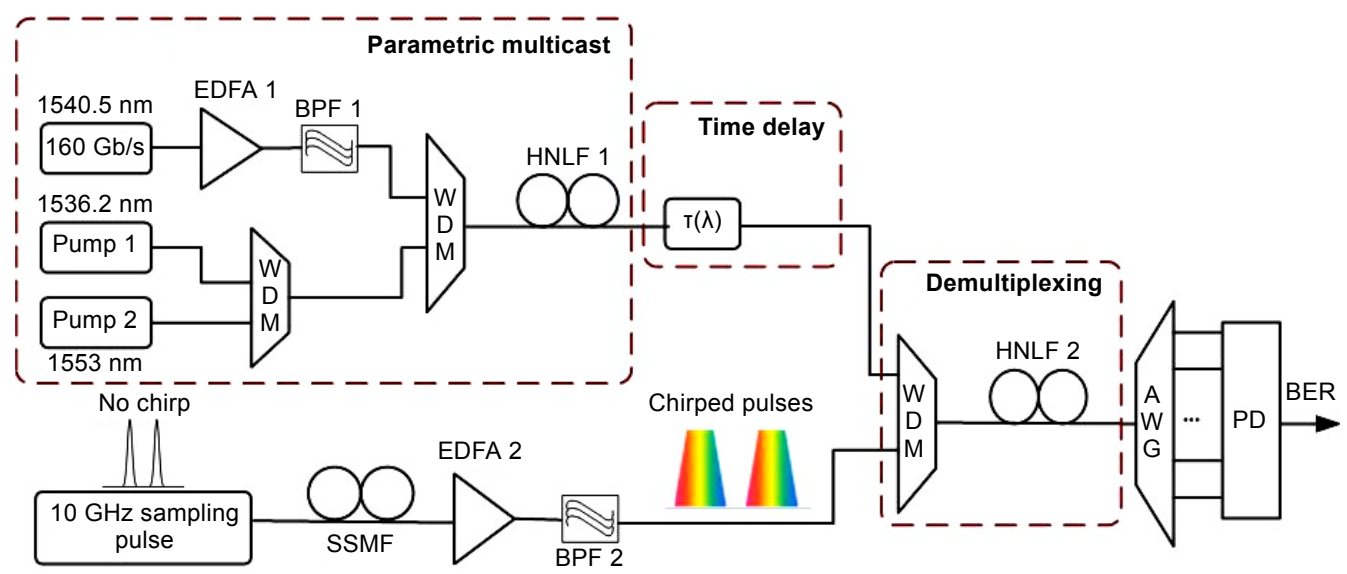

Fig. 4. Simulation set-up. EDFA - erbium-doped optical fiber amplifier, BPF - bandpass filter, WDM - wavelength division multiplexer, AWG - arrayed waveguide grating, SSMF - standard single-mode fiber, HNLF - high nonlinear fiber, PD - photoelectric detector, and BER - bit-error-rate. 

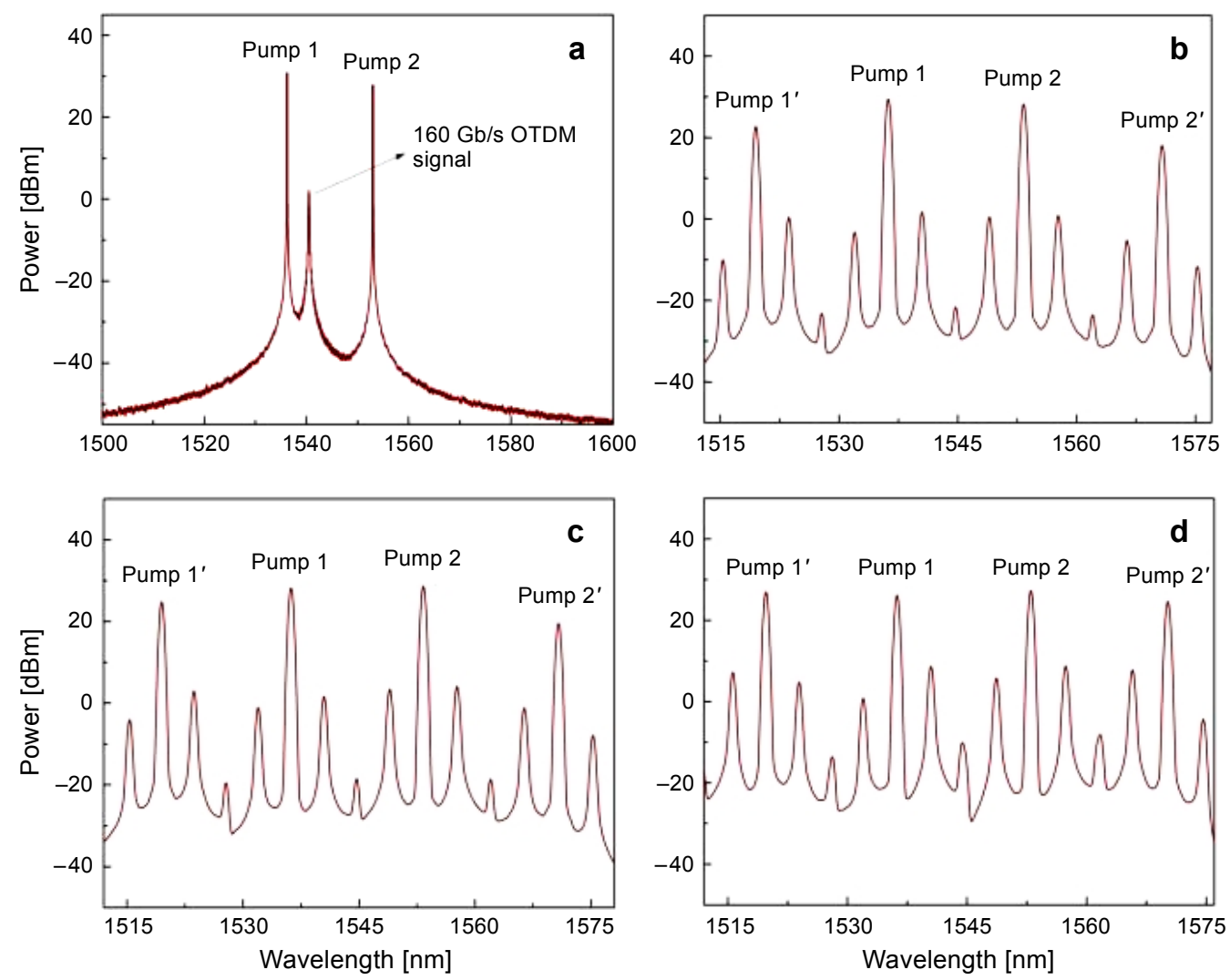

Fig. 5. The spectrum at the input of HNLF 1 (a). The spectrum at the output of HNLF 1 after the propagation of $30 \mathrm{~m} \mathrm{(b),} 50 \mathrm{~m} \mathrm{(c),} \mathrm{and} 90 \mathrm{~m}(\mathbf{d})$.

spectively, and adjusted carefully to generate the roughly equalized response. Figure 5a shows the spectrum at the input of the HNLF 1, composed of two pumps and the OTDM signal. As shown above, new set of pumps can be generated by taking advantage of the dual-pump FWM, which can eliminate the need of additional external pumps and decrease the complexity of the processor $[\underline{14}, \underline{16}, \underline{17}, \underline{19}]$. The pump 1' and pump 2' are spawned at 1519.7 and $1570.0 \mathrm{~nm}$, respectively. After the waves propagate inside the fiber, seven copies are generated by the interaction with the $160 \mathrm{~Gb} / \mathrm{s}$ OTDM signal at 1515.6, 1523.9, 1532.0, 1548.7, 1557.4, 1565.8, and $1574.6 \mathrm{~nm}$ (copies 1-3, 5-8), as shown in Figs. $5 \mathbf{b}, 5 \mathbf{c}$, and $5 \mathbf{d}$, respectively.

For the real-time MPASS processing, the time shift between the copies has to be set by a delay element positioned after the multicasting block. The time delay between each copy can achieve two bit periods, i.e. $6.25 \mathrm{ps}$ for the $160 \mathrm{~Gb} / \mathrm{s}$ rate, which ensures each $10 \mathrm{~Gb} / \mathrm{s}$ tributary to be mapped to a specific copy. Demultiplexing operation is believed to be possible not only by the generation of unimpaired data copies, each of 


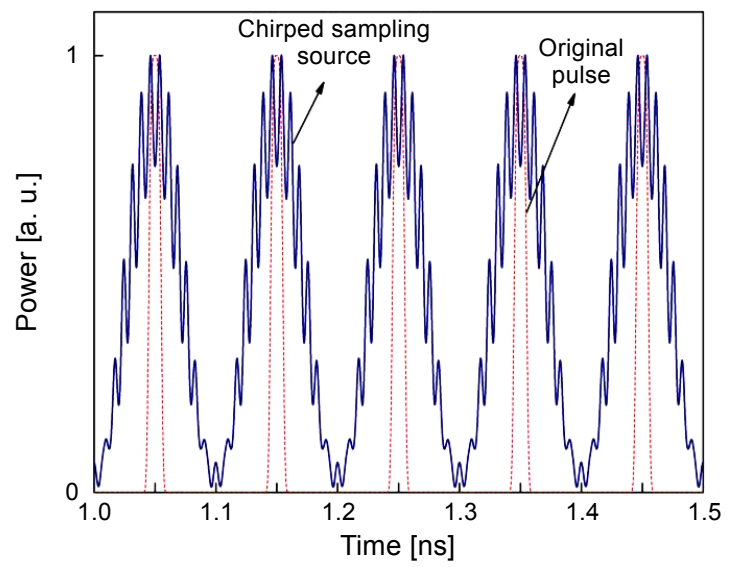

Fig. 6. The $10 \mathrm{GHz}$ linearly chirped pulses and time-broadened with the sampling source.

the eight copies located at a specific wavelength, but also by the broadened and linearly chirped pulses. The $10 \mathrm{GHz}$ linearly chirped sampling source (the blue solid line) is shown in Fig. 6 with the original pulses (the red dash line). It can be seen from Fig. 6 that the FWHM of the pulses is broadened from 5 to $50 \mathrm{ps.}$

The eight copies, which are generated by the parametric multicasting block, are coupled with the chirped pump source, and the pump ON/OFF operation of the block is shown in Fig. 7. The demultiplexing block maps every copy of $160 \mathrm{~Gb} / \mathrm{s}$ multicast channels (from 1515.0 to $1575.0 \mathrm{~nm}$ ) to its corresponding idler wavelengths (from 1594 to $1662 \mathrm{~nm}$ ). Then, the idlers are filtered by an AWG with the bandwidth of $0.2 \mathrm{~nm}$ at 16 wavelengths (channel 1-16: 1661.6, 1661.4, 1651.5, 1651.3, 1642.1, $1641.9,1632.3,1632.0,1623.1,1622.9,1613.5,1613.3,1604.4,1604.2,1594.3$, and

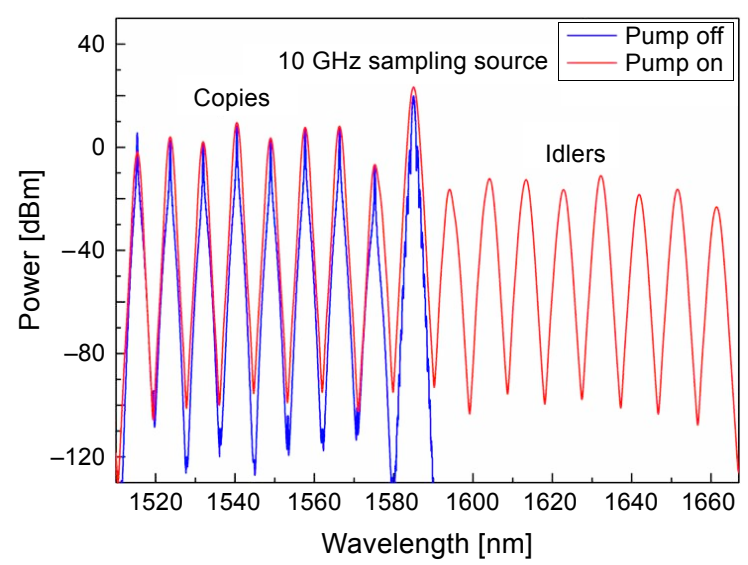

Fig. 7. Optical spectrum at the output of the HNLF 2 when the pump is on and off. 

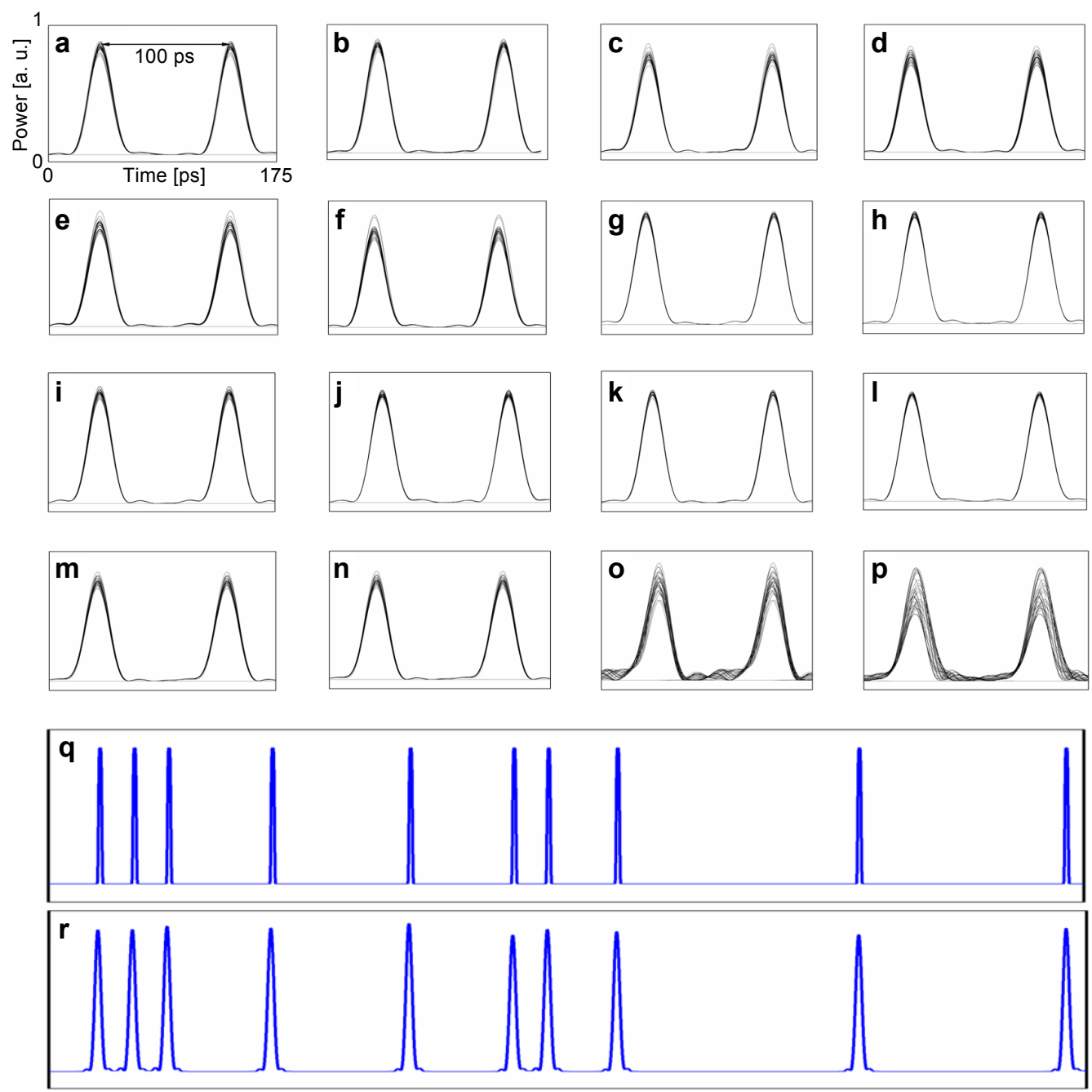

Fig. 8. Waveforms of recovered: corresponding to the sixteen channels' eye patterns recovered (a)-(p), the original $10 \mathrm{~Gb} / \mathrm{s}$ tributary $(\mathbf{q})$, and the recovered tributary of channel $1(\mathbf{r})$.

$1594.1 \mathrm{~nm}$, respectively). All sixteen recovered eye patterns are shown in Figs. $8 \mathbf{a}-8 \mathbf{p}$ along with the original $10 \mathrm{~Gb} / \mathrm{s}$ signal and recovered patterns on the sampled 1 (Figs. $8 \mathbf{q}$ and $8 \mathbf{r}$ ). Wide open eyes are observed with the excellent rejection of the adjacent tributaries. The change of the HNLF 1 length will induce the different gain ripples among the copies, and the power of the corresponding sampling points will also be different. As shown in Figs. $8 \mathbf{o}$ and 8p, the eye patterns of channel 15 and channel 16 are different from other channels since there is a bias in the powers between the copy 8 and other copies. In addition, the photodiodes are required at the output of the processor, so that the substrate outputs can be monitored simultaneously. Finally, the BER is measured on all sixteen demultiplexed channels, as shown in Fig. 9. Error-free perfor- 


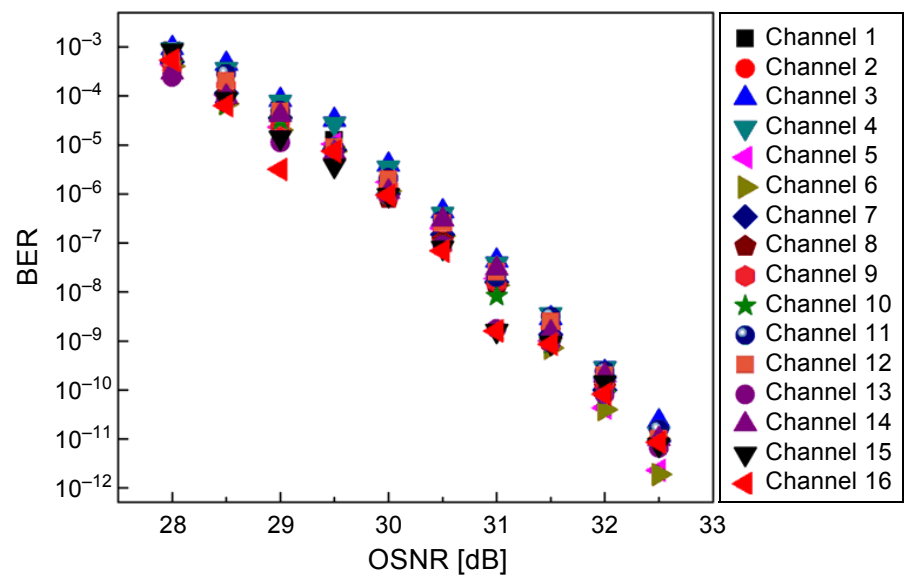

Fig. 9. BER measurements on all sixteen channels recovered.

mance of the demultiplexing block can reach the BER of $10^{-9}$, and the required OSNR is about $31.5 \mathrm{~dB}$. All demultiplexed channels have the similar changing tendency except for the channel 15 and channel 16, corresponding to the eye patterns of channel 15 and channel 16, as shown in Fig. $8 \mathbf{o}$ and $8 \mathbf{p}$. The recovery of all sixteen sampled streams at the output of the processor confirms that the high-quality data transfer to all eight multicast copies is achieved.

The powers of the pumps and copies are different at the output of the HNLF 1, which will induce the distortion of the demultiplexing. Thus, the power equalization of the eight copies is an important characteristic of the multicasting. The power evolutions for the pumps, the OTDM signal, and the copies along the HNLF 1 length are shown in Fig. 10. The representation of the multicasting process indicates that the generation of new pumps and copies takes place simultaneously inside a span of HNLF 1 . The powers of pump 1' and pump 2' grow within the first 20 m of HNLF 1. Copy 1 and copy 8

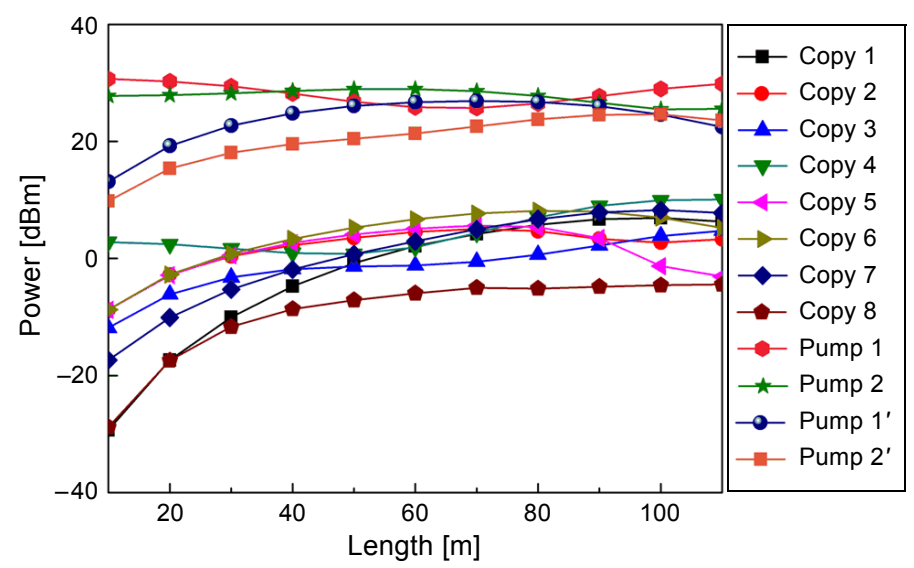

Fig. 10. Dependences of the pump and copy powers on the HNLF 1 length. 


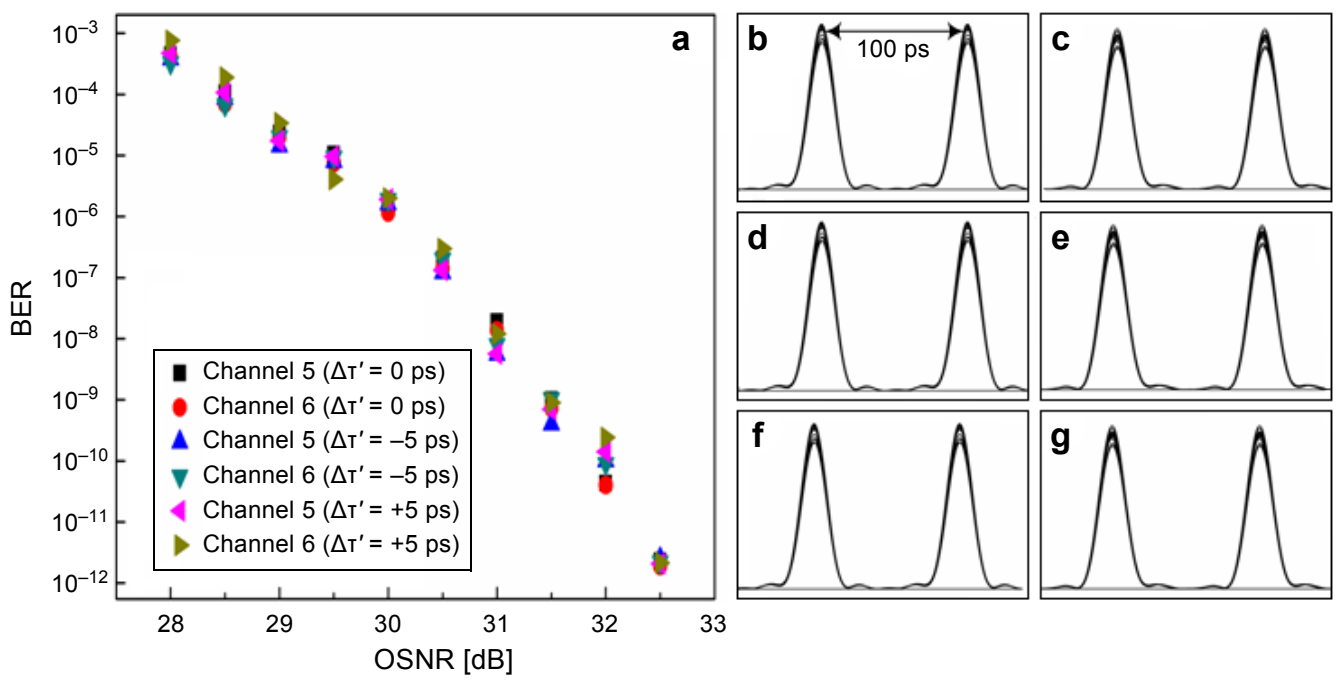

Fig. 11. BER measurements on the channels 5 and $6(\mathbf{a})$, the corresponding recovered eye patterns of the channels 5 and $6(\mathbf{b})-(\mathbf{e}) ; \Delta \tau^{\prime}=0,-5$, and +5 ps.

experience a slightly slower growth due to the strong dependences on the secondary pumps. The optimum length of HNLF 1 is $90 \mathrm{~m}$, the maximum power difference between the copies is about $13 \mathrm{dBm}$, and the power deviation among the copies is minimal. The equalized copies response coincides with the pump power equalization, as shown in Fig. 10.

The delay block is achieved by utilizing the tunable optical delay line, and the deviation of the temporal delay is another key factor, which has a negative influence on the demultiplexing. Here, we define the deviation between the actual and theoretical one as $\Delta \tau^{\prime}=\Delta \tau-\Delta \tau_{\text {actual }}$. Taking the copy 3 for example in our simulation, when $\Delta \tau^{\prime}=0$, -5 , and +5 ps, the BERs of channel 5 and channel 6 are shown in Fig. 11a, and the corresponding recovered eye patterns are shown in Figs. 11b-11g. From Fig. 11a, the BERs of channel 5 and channel 6 will be changed when $\Delta \tau^{\prime}$ is changed, along with a similar change tendency. The wide open eyes are observed with different $\Delta \tau^{\prime}$, as shown in Figs. $11 \mathbf{b}$ and $11 \mathbf{c}$. And the deviation of the temporal delay can be neglected. Therefore, the performance of the proposed scheme with different parameters is necessary to be evaluated to obtain the optimized demultiplexing result.

\section{Conclusion}

In summary, the optical deconstruction of a $160 \mathrm{~Gb} / \mathrm{s}$ OTDM signal with simultaneous generation of all sixteen $10 \mathrm{~Gb} / \mathrm{s}$ tributaries is demonstrated. By cascading a parametric multicasting block and a parametric synchronous sampling block, the parametric multicast block, which is achieved by a dual-pump parametric amplifier, is used to generate the eight spectral replicas of the $160 \mathrm{~Gb} / \mathrm{s}$ OTDM signal. The parametric demultiplexing 
is realized by using the time-broadened and linearly chirped pulses. The error-free performance on all extracted $10 \mathrm{~Gb} / \mathrm{s}$ tributaries can be shown by the BER measurement. It is believed that the proposed scheme can find important applications in the high-speed all-optical signal processing.

Acknowledgments - This work is partly supported by the National Natural Science Foundation of China (61307109 and 61475023), the Natural Science Foundation of Beijing (4152037), the National High -Technology Research and Development Program of China (2013AA031501), the Specialized Research Fund for the Doctoral Program of Higher Education (20120005120021), the Fundamental Research Funds for the Central Universities (2013RC1202), the Fund of State Key Laboratory of Information Photonics and Optical Communications (Beijing University of Posts and Telecommunications) P.R. China, the Hong Kong Scholars Program 2013 (PolyU G-YZ45), and the Research Grant Council of the Hong Kong Special Administrative Region China (PolyU5272/12E).

\section{References}

[1] Bhushan A.S., Coppinger F., Jalali B., Time-stretched analogue-to-digital conversion, Electronics Letters 34(9), 1998, pp. 839-841.

[2] Hao Chi, Ying Chen, Yuan Mei, Xiaofeng Jin, Shilie Zheng, Xianmin Zhang, Microwave spectrum sensing based on photonic time stretch and compressive sampling, Optics Letters 38(2), 2013. pp. 136-138.

[3] Hao Nan, Yuantao Gu, Hongming Zhang, Optical analog-to-digital conversion system based on compressive sampling, IEEE Photonics Technology Letters 23(2), 2011, pp. 67-69.

[4] Airola M.B., O’Connor S.R., Dennis M.L., Clark T.R., Experimental demonstration of a photonic analog-to-digital converter architecture with pseudorandom sampling, IEEE Photonics Technology Letters 20(24), 2008, pp. 2171-2173.

[5] Zhe Kang, Jinhui Yuan, Qiang Wu, Tao Wang, Sha Li, Xinzhu Sang, Chongxiu Yu, Farrell G., Lumped time-delay compensation scheme for coding synchronization in the nonlinear spectral quantization-based all-optical analog-to-digital conversion, IEEE Photonics Journal 5(6), 2013, article ID 7201109.

[6] Kang Zhe, Yuan Jin-Hui, Li Sha, Xie Song-Lin, Yan Bin-Bin, SAng Xin-Zhu, Yu Chong-Xiu, Six-bit all-optical quantization using photonic crystal fiber with soliton self-frequency shift and pre-chirp spectral compression techniques, Chinese Physics B 22(11), 2013, article ID 114211.

[7] Dingkang Tang, Jianguo Zhang, Yuanshan Liu, Wei Zhao, Ultrashort optical pulse monitoring using asynchronous optical sampling technique in highly nonlinear fiber, Chinese Optics Letters 8(7), 2010, pp. 630-633.

[8] Westlund M., Andrekson P.A., Sunnerud H., Hansryd J., Jie Li, High-performance optical-fiber -nonlinearity-based optical waveform monitoring, Journal of Lightwave Technology 23(6), 2005, pp. 2012-2022.

[9] Hedekvist P.O., Karlsson M., Andrekson P.A., Fiber four-wave mixing demultiplexing with inherent parametric amplification, Journal of Lightwave Technology 15(11), 1997, pp. 2051-2058.

[10] Jansen S.L., Heid M., Spalter S., Meissner E., Weiske C.-J., Schopflin A., Khoe D., De WaArdt H., Demultiplexing $160 \mathrm{Gbit} / \mathrm{s}$ OTDM signal to $40 \mathrm{Gbit} / \mathrm{s}$ by FWM in SOA, Electronics Letters 38(17), 2002, pp. 978-980.

[11] Rau L., Wei Wang, Olsson B.-E., Yijen Chiu, Hsu-Feng Chou, Blumenthal D.J., Bowers J.E., Simultaneous all-optical demultiplexing of a 40-Gb/s signal to $4 \times 10 \mathrm{~Gb} / \mathrm{s}$ WDM channel's using an ultrafast fiber wavelength converter, IEEE Photonics Technology Letters 14(12), 2002, pp. 1725 -1727 . 
[12] Palushani E., Hansen Mulvad H.C., Galili M., Hao Hu, Oxenlowe L.K., Clausen A.T., Jeppesen P., OTDM-to-WDM conversion based on time-to-frequency mapping by time-domain optical Fourier transformation, IEEE Journal of Selected Topics in Quantum Electronics 18(2), 2012, pp. 681-688.

[13] Wiberg A.O.J., Bres C.-S., Windmiller J.R., Alic N., Radic S., RZ pulse source for optical time division multiplexing based on self-phase modulation and four wave mixing, 2009 IEEE/LEOS Winter Topicals Meeting Series, 2009, pp. 233-234.

[14] Bres C.-S., Wiberg A.O.J., Kuo B.P.-P., Chavez-Boggio J.M., Marki C.F., Alic N., Radic S., Optical demultiplexing of $320 \mathrm{~Gb} / \mathrm{s}$ to $8 \times 40 \mathrm{~Gb} / \mathrm{s}$ in single parametric gate, Journal of Lightwave Technology 28(4), 2010, pp. 434-442.

[15] Bres C.-S., Wiberg A.O.J., Kuo B.P.-P., Chavez-Boggio J.M., Marki C.F., Alic N., Radic S., Single-gate 320-to- $8 \times 40 \mathrm{~Gb} / \mathrm{s}$ demultiplexing, Optical Fiber Communication Conference, Optical Society of America, 2009, article ID PDPA4.

[16] Bres C.-S., Wiberg A.O.J., Kuo B.P.-P., Alic N., Radic S., Wavelength multicasting of 320-Gb/s channel in self-seeded parametric amplifier, IEEE Photonics Technology Letters 21(14), 2009, pp. 1002-1004.

[17] Bres C.-S., Alic N., Gnauck A.H., Jopson R.M., Radic S., Multicast parametric synchronous sampling, IEEE Photonics Technology Letters 20(14), 2008, pp. 1222-1224.

[18] Xianting Zhang, Zhe Kang, Jinhui Yuan, Qiang Wu, Farrell G., Xinzhu Sang, Chongxiu Yu, Scheme for multicast parametric synchronous optical sampling, Optical Engineering 53(5), 2014, article ID 056102.

[19] Bres C.-S., Wiberg A.O.J., Windmiller J.R., Alic N., Radic S., Parametric multicasting of $320 \mathrm{~Gb} / \mathrm{s}$ OTDM data, 2009 IEEE/LEOS Winter Topicals Meeting Series, 2009, pp. 193-194.

[20] McKinstrie C.J., Radic S., Chraplyvy A.R., Parametric amplifiers driven by two pump waves, IEEE Journal of Selected Topics in Quantum Electronics 8(3), 2002, pp. 538-547.

[21] Salem R., Foster M.A., Turner-Foster A.C., Geraghty D.F., Lipson M., Gaeta A.L., High-speed optical sampling using a silicon-chip temporal magnifier, Optics Express 17(6), 2009, pp. 4324-4329.

[22] Agrawal G.P., Nonlinear Fiber Optics, Academic Press, 2007.

[23] Salem R., Foster M.A., Turner A.C., Geraghty D.F., Lipson M., Gaeta A.L., Optical time lens based on four-wave mixing on a silicon chip, Optics Letters 33(10), 2008, pp. 1047-1049.

[24] Hansen Mulvad H.C., Palushani E., Hao Hu, Hua Ji, Lillieholm M., Galili M., Clausen A.T., Minhao Pu, Yvind K., Hvam J.M., Jeppesen P., Oxenløwe L.K., Ultra-high-speed optical serial-to -parallel data conversion by time-domain optical Fourier transformation in a silicon nanowire, Optics Express 19(26), 2011, pp. B825-B835.

Received September 13, 2017

in revised form January 12, 2018 